\title{
A SYSTEM OF ORDINARY LINEAR DIFFERENTIAL EQUATIONS WITH TWO-POINT BOUNDARY CONDITIONS*
}

\author{
BY \\ WILLIAM T. REID
}

1. Introduction. In a recent paper Bliss [2] $\dagger$ has treated a boundary value problem which is definitely self-adjoint according to a weakened form of an earlier definition of definite self-adjointness which he gave in a paper [2] published in 1926. He has shown that a system which satisfies this modified definition has most of the properties proved in the former paper for a system satisfying the original and stronger definition of definite self-adjointness. The characteristic values for such a problem are all real and have indices equal to their multiplicities, and the expansion theorems established in the earlier paper are still valid. Such a system, however, will not in general have an infinity of characteristic values.

Bliss has shown that the canonical form of the so-called accessory boundary value problem for a normal non-singular problem of Bolza in the calculus of variations is definitely self-adjoint according to this new definition, while such a problem is in general not definitely self-adjoint according to the original definition. $\mathrm{Hu}$ [5] has proved that this problem has an infinity of characteristic values. Various authors (Morse [7] and [8], Reid [9], [10], and [11], Hu [5], Hölder [4], Birkhoff and Hestenes [1], Wiggin [12]) have treated the accessory problem under the assumptions that the problem is normal and satisfies the strengthened Clebsch condition. This latter condition implies non-singularity. Under these hypotheses the characteristic values possess certain extremizing properties. Moreover, for such systems there have been established oscillation and comparison theorems which are generalizations of the classic results of the Sturmian theory for a single second order differential equation (see, for example, Ince [6], chap. 10). It is to be remarked that for the problem treated by the author ([9] and [10]) it is not assumed that the coefficients of the terms involving the parameter are those of a definite quadratic form. To compensate for this weakened condition it it assumed that the quadratic functional of the associated minimum problem is definite. This latter hypothesis is no additional restriction for an accessory problem which is normal, satisfies the strengthened Clebsch condition, and

* Presented to the Society, April 11, 1936; received by the editors November 3, 1937.

$\dagger$ Numerals in square brackets refer to the bibliography at the end of the present paper. 
for which the coefficients of the terms involving the parameter belong to a definite quadratic form. In the author's paper [9] there are also established expansion theorems in terms of the characteristic solutions of the problem.

It is the purpose of the present paper to consider a boundary problem which is definitely self-adjoint according to the new definition of Bliss, and which satisfies the additional condition that the matrix of coefficients of the terms involving the parameter has constant rank on the interval of definition. This latter condition is satisfied in the most important examples of definitely. self-adjoint systems. It is here shown that such a system is equivalent to a boundary value problem associated with the second variation of a calculus of variations problem, and of the type previously treated by the author [9]. The character of the equivalence is new in the sense that the canonical form of the second problem is not the given definitely self-adjoint problem, but rather a system involving twice the number of dependent functions occurring in the original system. There is obtained a general condition which is both necessary and sufficient for the given system to have an infinity of characteristic values. There are also given two different sets of conditions which are merely sufficient for this conclusion. In particular, in $\$ 5$ it is shown that an accessory boundary value problem which is normal and non-singular has an infinity of characteristic values. As stated above, this result was first proved by $\mathrm{Hu}$. As already noted, oscillation, comparison, and expansion theorems are known for a system of the form to which the given definitely self-adjoint problem is here shown to be equivalent. From these theorems there are deduced in $\$ 6$ certain corresponding results for the given boundary value problem.

The notation used for the definitely self-adjoint system here treated is the same as that used by Bliss in papers [2] and [3]. Frequent references are made to the results concerning differential systems in general, and the theorems for self-adjoint systems, obtained by Bliss in $\$ \$ 1$ and 2 of paper [2]. It is to be noted that for the particular definitely self-adjoint problem herein treated the proof of the reality of characteristic values is independent of the proof of paper [3] of Bliss, since this result is here deduced from the corresponding theorem for the equivalent problem of the type previously treated by the author.

2. Statement of problem. In the following pages the summation convention of tensor analysis will be used. The subscripts $i, j, k$ will have the range $1, \cdots, n$. The matrices $\left\|A_{i j}(x)\right\|,\left\|B_{i j}(x)\right\|$ will be supposed to have elements which are real-valued and continuous on $a \leqq x \leqq b$, while the elements of the matrices $\left\|M_{i j}\right\|,\left\|N_{i j}\right\|$ are real constants such that the matrix $\left\|M_{i j} ; N_{i j}\right\|$ has rank $n$. For brevity we will introduce the notations*

\footnotetext{
* Here, as elsewhere, primes are used to denote derivatives with respect to the variable $x$.
} 


$$
\mathcal{L}_{i}[y]=y_{i}^{\prime}-A_{i j}(x) y_{j}, \quad \mathscr{X}_{i}[z]=z_{i}^{\prime}+z_{j} A_{j i}(x) .
$$

The boundary value problem to be considered in this paper may then be written as

$$
\mathcal{L}_{i}[y]=\lambda B_{i j}(x) y_{j}, \quad s_{i}[y] \equiv M_{i j} y_{j}(a)+N_{i j} y_{j}(b)=0 .
$$

The system adjoint to (2.2) is (see Bliss [2]),

$$
\mathcal{X}_{i}[z]=-\lambda z_{j} B_{j i}(x), \quad t_{i}[z]=z_{j}(a) P_{j i}+z_{j}(b) Q_{j i}=0,
$$

where $p_{i}=P_{i j}, q_{i}=Q_{i j},(j=1, \cdots, n)$, are linearly independent solutions of the algebraic equations

$$
M_{i j} p_{i}-N_{i j} q_{j}=0 .
$$

The boundary value problem (2.2) will be treated under the following hypotheses:

$\left(\mathrm{H}_{1}\right)$ The system (2.2) is self-adjoint under the non-singular transformation $z_{i}=T_{i j}(x) y_{i}$, that is, under this transformation the differential equations and boundary conditions of the adjoint system (2.3) are equivalent to those of (2.2) for all values of $\lambda$. The elements $T_{i j}(x)$ are supposed to be of class $C^{1}$ on the interval $a b$.

A non-singular matrix $\left\|T_{i j}(x)\right\|$ of functions of class $C^{1}$ is such a transformation matrix if and only if

$$
\begin{gathered}
T_{i k} A_{k j}+A_{k i} T_{k j}+T_{i j}^{\prime} \equiv 0, \quad T_{i k} B_{k j}+B_{k i} T_{k j} \equiv 0, \\
M_{i k} T_{k l}^{-1}(a) M_{j l}=N_{i k} T_{k l}^{-1}(b) N_{j l} .
\end{gathered}
$$

In particular, if $u_{i}(x)$ and $v_{i}(x)$ are sets of functions of class $C^{1}$ and are related by the equations $v_{i}(x) \equiv T_{j i}(x) u_{j}(x)$, we have the identity

$$
\mathscr{x}_{i}[v] \equiv T_{j i}(x) \mathcal{L}_{i}[u] \text {. }
$$

Moreover, $t_{i}[v]=0$ if and only if $s_{i}[u]=0$.

$\left(\mathrm{H}_{2}\right)\left\|S_{i j}(x)\right\| \equiv\left\|T_{k i}(x) B_{k j}(x)\right\|$ is symmetric.

$\left(\mathrm{H}_{3}\right)$ If $\left(u_{i}\right)$ is a set of real constants, then

$$
u_{i} S_{i j}(x) u_{i} \geqq 0 .
$$

$\left(\mathrm{H}_{4}\right)$ There exists no non-identically vanishing solution of $\mathcal{L}_{i}[y]=0$, $s_{i}[y]=0$ such that $B_{i j}(x) y_{j}(x) \equiv 0$ on $a b$.

If the system (2.2) is the canonical form of a so-called accessory boundary value problem associated with the second variation of a problem of the calculus of variations, the above hypothesis $\left(\mathrm{H}_{4}\right)$ is equivalent to the assumption 
of normality of the accessory problem. The significance of this hypothesis for the general self-adjoint system of the form (2.2) has been pointed out by Bliss [3].

$\left(\mathrm{H}_{5}\right)\left\|B_{i j}(x)\right\|$ is of constant rank $n-m,(0 \leqq m<n)$, on $a \leqq x \leqq b$.

If $y_{i}(a), y_{i}(b), z_{i}(a), z_{i}(b)$ are arbitrary values, we have (Bliss [2]),

$$
\left.y_{i}(x) z_{i}(x)\right|_{a} ^{b}=\bar{t}_{i}[z] s_{i}[y]+t_{i}[z] \bar{s}_{i}[y],
$$

where $\bar{s}_{i}[y]=\bar{M}_{i j} y_{j}(a)+\bar{N}_{i j} y_{j}(b), \bar{t}_{i}[z]=z_{j}(a) \bar{P}_{j i}+z_{j}(b) \bar{Q}_{j i}$, and the matrices $\left\|\bar{M}_{i j}\right\|,\left\|\bar{N}_{i j}\right\|,\left\|\bar{P}_{i j}\right\|,\left\|\bar{Q}_{i j}\right\|$ are chosen so that

$$
\left\|\begin{array}{ll}
M_{i j} & N_{i j} \\
\bar{M}_{i j} & \bar{N}_{i j}
\end{array}\right\|, \quad\left\|\begin{array}{rr}
-\bar{P}_{i j} & -P_{i j} \\
\bar{Q}_{i j} & Q_{i j}
\end{array}\right\|
$$

are reciprocals. Moreover, if $y_{i}(x), z_{i}(x)$ are solutions of the differential equations of the systems (2.2) and (2.3), respectively, for some value of $\lambda$, then $\left[y_{i}(x) z_{i}(x)\right]^{\prime}=0$. Combining these results, and making use of the self-adjointness of the system (2.2) under the transformation $z_{i}=T_{i j} y_{j}$, we obtain that if $y_{i}(x)$ and $y_{i}^{*}(x)$ are solutions of (2.2) corresponding to distinct values $\lambda$ and $\lambda^{*}$, respectively, then (see Bliss [2])

$$
\int_{a}^{b} y_{i}(x) S_{i j}(x) y_{j}^{*}(x) d x=0 .
$$

3. Relation of the boundary value problem to the calculus of variations. In this section there will be pointed out the relation of the above boundary value problem (2.2) with a problem of the form of an accessory boundary problem associated with the calculus of variations.

As a consequence of hypotheses $\left(\mathrm{H}_{2}\right),\left(\mathrm{H}_{3}\right)$, and $\left(\mathrm{H}_{5}\right)$ there exist $m$ sets of functions $\pi_{i \alpha}(x),(\alpha=1, \cdots, m)$, such that

$$
S_{i j}(x) \pi_{j \alpha}(x) \equiv 0, \quad i=1, \cdots, n ; \alpha=1, \cdots, m ; a \leqq x \leqq b .
$$

Moreover, these functions $\pi_{i \alpha}(x)$ may be chosen as continuous on $a \leqq x \leqq b$, and orthonormal in the sense that

$$
\pi_{i \alpha}(x) \pi_{i \beta}(x)=\delta_{\alpha \beta}, \quad \alpha, \beta=1, \cdots, m .
$$

In view of $\left(\mathrm{H}_{3}\right)$ it follows that the matrix

$$
\left\|\begin{array}{ll}
S_{i j}(x) & \pi_{i \beta}(x) \\
\pi_{j \alpha}(x) & 0_{\alpha \beta}
\end{array}\right\|
$$

is non-singular on $a b$. The reciprocal of this matrix is seen to be of the form 


$$
\left\|\begin{array}{ll}
R_{i j}(x) & \pi_{i \beta}(x) \\
\pi_{j \alpha}(x) & 0_{\alpha \beta}
\end{array}\right\|
$$

and $R_{i j}(x) u_{i} u_{j}>0$ for every set $\left(u_{i}\right) \neq\left(0_{i}\right)$ satisfying the relations $\pi_{j \alpha}(x) u_{i}=0$, $(\alpha=1, \cdots, m)$.

Finally, we shall suppose that there are exactly $\rho$ linearly independent solutions $y_{i}=u_{i \kappa}(x),(\kappa=1, \cdots, \rho)$, of the system (2.2) for $\lambda=0$. Bliss has shown that under the hypotheses $\left(\mathrm{H}_{1}\right)-\left(\mathrm{H}_{4}\right)$ the index of a characteristic value is equal to its multiplicity as a root of the characteristic equation $D(\lambda)=0$. Hence not all values of $\lambda$ can be characteristic values of (2.2) under these hypotheses, and without loss of generality we might assume $\lambda=0$ is not a characteristic value. The method of proof as given by Bliss is similar to that used in his previous paper [2] to establish the same result under stronger hypotheses. However, for completeness and independence of the results of the present paper, use will not be made of this result. It is to be pointed out that Theorem 3.1 below includes the result that under hypotheses $\left(\mathrm{H}_{1}\right)-\left(\mathrm{H}_{6}\right)$ all values of $\lambda$ cannot be characteristic values of (2.2).

An $\operatorname{arc} z \equiv\left[z_{i}(x)\right]$ will be called admissible if the functions $z_{i}(x)$ are of class $D^{\prime}$ on $a b$, and satisfy the differential equations

$$
\pi_{j \alpha}(x) \mathscr{X}_{j}[z]=0,
$$

$$
\alpha=1, \cdots, m \text {. }
$$

For brevity, the class $H_{1}^{*}$ will be defined as the totality of admissible arcs $z$ satisfying the conditions

$$
\begin{array}{ll}
t_{i}[z] \equiv z_{j}(a) P_{j i}+z_{j}(b) Q_{j i}=0, & i=1, \cdots, n, \\
\int_{a}^{b} z_{i}(x) B_{i j}(x) u_{j \kappa}(x) d x=0, & \kappa=1, \cdots, \rho, \\
\int_{a}^{b} z_{i}(x) K_{i j}(x) z_{j}(x) d x=1, &
\end{array}
$$

where $K_{i j}(x)=-B_{i k}(x) T_{j k}^{-1}(x)$. Since $K_{i j}(x)=T_{i k}^{-1}(x) S_{k l}(x) T_{j l}^{-1}(x)$, the matrix $\left\|K_{i j}(x)\right\|$ is symmetric and $u_{i} K_{i j}(x) u_{j} \geqq 0$ for all real sets $\left(u_{i}\right) \neq\left(0_{i}\right)$.

Suppose the class $H_{1}^{*}$ is non-vacuous, and consider the problem of minimizing the integral

$$
I[z]=\int_{a}^{b} \mathscr{H}_{i}[z] R_{i j}(x) \mathscr{X}_{i}[z] d x
$$

in this class. In view of (3.4) we have that $I[z]$ is non-negative in the class of admissible arcs.

Because of the integral conditions (3.6) this minimum problem is not of 
the form of an accessory problem associated with the problem of Bolza in the calculus of variations. It may be reduced, however, to such a problem by the introduction of new variables $z_{n+\kappa}$ satisfying the differential equations $z_{n+\kappa}^{\prime}-z_{i} B_{i j} u_{j \kappa}=0$ and end conditions $z_{n+\kappa}(a)-z_{n+\kappa}(b)=0$.

If, for brevity, we set

$$
R_{i j}(x) \mathcal{H}_{j}[z]+\pi_{i \alpha}(x) \mu_{\alpha}(x)=\zeta_{i}(x),
$$

the first necessary condition for this calculus of variations problem states that there exist multipliers $\mu_{\alpha}(x)$ and constants $\sigma_{\kappa}, \Lambda, d_{i}$, such that in addition to equations (3.4), (3.5), (3.6), (3.7) we have

$$
\begin{gathered}
\zeta_{i}^{\prime}-A_{i j} \zeta_{j}-B_{i j} u_{j \kappa} \sigma_{\kappa}+\Lambda K_{i j} z_{j}=0, \\
P_{i j} d_{j}-\zeta_{i}(a)=Q_{i j} d_{j}+\zeta_{i}(b)=0,
\end{gathered}
$$

where $\zeta_{i}(x)$ is the function defined in terms of $z_{i}(x), \mu_{\alpha}(x)$ by (3.9). Solving (3.4) and (3.9) simultaneously, we obtain

$$
\mathscr{X}_{i}[z]=S_{i j}(x) \zeta_{i}, \quad \mu_{\alpha}(x)=\pi_{j \alpha}(x) \zeta_{j} .
$$

Moreover, since $M_{i k} P_{k j}-N_{i k} Q_{k j}=0$, the equations (3.11) are equivalent to the conditions $s_{i}[\zeta]=0$. Consequently, the system (3.4), (3.5), (3.6), (3.10), (3.11) may be written

$$
\begin{aligned}
& \mathcal{X}_{i}[z]=S_{i j}(x) \zeta_{j}, \quad \mathcal{L}_{i}[\zeta]=B_{i j} u_{j \kappa} \sigma_{\kappa}-\Lambda K_{i j} z_{j}, \\
& \int_{a}^{b} z_{i}(x) B_{i j}(x) u_{j \kappa}(x) d x=0, \quad t_{i}[z]=0, \quad s_{i}[\zeta]=0, \\
& i, j=1, \cdots, n ; \kappa=1, \cdots, \rho .
\end{aligned}
$$

Now, since $y_{i}=u_{i \kappa}(x),(\kappa=1, \cdots, \rho)$, is a solution of $\mathcal{L}_{i}[y]=0, s_{i}[y]=0$, we have that $z_{i}=v_{i \kappa}(x)=T_{j i}(z) u_{j \kappa}(x)$ is a solution of $\mathcal{X}_{i}[z]=0, t_{i}[z]=0$. Using this relation, together with (2.6), we obtain in view of $\left(\mathrm{H}_{3}\right)$ and $\left(\mathrm{H}_{4}\right)$ the following result:

Lemma 3.1. If $z_{i}, \zeta_{i}$ satisfies (3.12) with constants $\sigma_{\kappa}, \Lambda$, then $\sigma_{\kappa}=0$, $(\kappa=1, \cdots, \rho)$.

LEMMA 3.2. The system (3.12) is normal.

For if this system is not normal, there exist functions $z_{i}(x) \equiv 0$, $\zeta_{i}(x) \not \equiv 0$ satisfying with constants $\sigma_{\kappa}, \Lambda$ this system. In view of Lemma 3.2, $\sigma_{\kappa}=0$. Then $\zeta_{i} \equiv \pi_{i \alpha}(x) \mu_{\alpha}(x)$ is a solution of $\mathcal{L}_{i}[\zeta]=0, s_{i}[\zeta]=0$. But since $B_{i j} \pi_{j \alpha} \equiv 0$, we then have $B_{i j}(x) \zeta_{j} \equiv 0$ and by $\left(\mathrm{H}_{4}\right)$ we have $\zeta_{i}(x) \equiv 0$, contrary to the assumption that $\zeta_{i}(x) \not \equiv 0$. Therefore the system (3.12) is normal.

Lemma 3.3. The value $\Lambda=0$ is not a characteristic value of (3.12). 
This result is a consequence of Lemma 3.1, relation (2.6), hypotheses $\left(\mathrm{H}_{3}\right)$ and $\left(\mathbf{H}_{4}\right)$, together with the fact that every solution of the system $\mathcal{L}_{i}[y]=0$, $s_{i}[y]=0$ is a linear combination of the functions $u_{i \kappa}(x),(\kappa=1, \cdots, \rho)$.

THEOREM 3.1. The characteristic values $\Lambda$ of (3.12) are all real and positive, and at most denumerably infinite in number.

The reality of the characteristic values has been proved by the author [9]. The positiveness is a consequence of the above hypotheses and the identity

$$
I[z]=\Lambda \int_{a}^{b} z_{i}(x) K_{i j}(x) z_{j}(x) d x
$$

which is satisfied by the functions $z_{i}(x)$ of a solution $z_{i}, \zeta_{i}, \sigma_{k}=0$ of (3.12) for a corresponding value $\Lambda$. Since $\Lambda=0$ is not a characteristic value, and the characteristic values are the zeros of a permanently convergent power series (see Bliss [2]), we have at most a denumerable infinity of such values.

The following properties have been proved by Reid [9]:

THEOREM 3.2. If the class $H_{1}^{*}$ is non-vacuous and $\Lambda_{1}$ is the greatest lower bound of $I[z]$ in this class, then $\Lambda_{1}>0$ and $\Lambda=\Lambda_{1}$ is the least characteristic value of (3.12).

TheOREM 3.3. Suppose $\Lambda_{1}<\Lambda_{2}<\cdots<\Lambda_{t-1}$ are consecutive characteristic values of (3.12), and corresponding to $\Lambda=\Lambda_{s},(s=1, \cdots, t-1)$, there are $r_{s}$ linearly independent solutions $z_{i q_{s}}, \zeta_{i q_{s}}, \sigma_{\kappa q_{s}}=0,\left(q_{s}=1, \cdots, r_{s}\right)$. Define the class $H_{t}^{*}$ as the subclass of arcs $z$ belonging to $H_{1}^{*}$ and satisfying the additional conditions

$$
0=\int_{a}^{b} z_{i}(x) K_{i j}(x) z_{j q_{s}}(x) d x, \quad q_{s}=1, \cdots, r_{s} ; s=1, \cdots, t-1 .
$$

If $H_{t}^{*}$ is non-vacuous and $\Lambda_{t}$ is the greatest lower bound of $I[z]$ in this class, then $\Lambda_{t}>\Lambda_{t-1}$ and $\Lambda=\Lambda_{t}$ is a characteristic value of (3.12).

If $z_{i}, \zeta_{i}, \sigma_{k}=0$ is a solution of (3.12) for a value $\Lambda$, set $z_{i}=\left(1 / \Lambda^{1 / 2}\right) T_{j i}(x) \eta_{j}$. Then $\eta_{i}, \zeta_{i}$ is a solution of the system

$$
\mathcal{L}_{i}[\eta]=\Lambda^{1 / 2} B_{i j}(x) \zeta_{j}, \quad \mathcal{L}_{i}[\zeta]=\Lambda^{1 / 2} B_{i j}(x) \eta_{j}, \quad s_{i}[\eta]=0, \quad s_{i}[\zeta]=0 .
$$

Now if $\bar{\eta}_{i}, \bar{\zeta}_{i}$ is a solution of (3.13), the functions $\eta_{i}=\bar{\eta}_{i}+\bar{\zeta}_{i}, \zeta_{i}=\bar{\eta}_{i}+\zeta_{i}$ and $\eta_{i}=\bar{\eta}_{i}-\bar{\zeta}_{i}, \zeta_{i}=\bar{\zeta}_{i}-\bar{\eta}_{i}$ are also solutions of this system. Consequently, if the index of $\Lambda$ as a characteristic value of (3.12) is equal to $r$, there exist $r$ linearly independent solutions $\eta_{i}, \zeta_{i}$ of (3.13) such that for each of these solutions we have either $\eta_{i}=\zeta_{i}$ or $\eta_{i}=-\zeta_{i}$. Now if $\eta_{i}, \zeta_{i}=\eta_{i}$ is a characteristic solution of (3.13) we see that $y_{i}=\eta_{i}$ is a characteristic solution of (2.2) for $\lambda=\Lambda^{1 / 2}$; simi- 
larly, if $\eta_{i}, \zeta_{i}=-\eta_{i}$ is a characteristic solution of (3.13), we have that $y_{i}=\eta_{i}$ is a solution of (2.2) for $\lambda=-\Lambda^{1 / 2}$. Hence the sum of the indices of $\Lambda^{1 / 2}$ and $-\Lambda^{1 / 2}$ as characteristic values of (2.2) is not less than the index of $\Lambda$ as a characteristic value of (3.12).

Now if $\lambda \neq 0$ is a characteristic value of (2.2) and $y_{i}$ is a corresponding characteristic solution, then $z_{i}=(1 / \lambda) T_{j i} y_{j}, \zeta_{i}=y_{i}$ is a solution of (3.12) for $\Lambda=\lambda^{2}$. The orthogonality of the functions $z_{i}(x)$ to the sets $u_{i \kappa}(x),(\kappa=1, \cdots, \rho)$, is a consequence of (2.7). In view of Lemma 3.1 we have, therefore, that all the characteristic values of (2.2) are real. In view of (2.7) the set of characteristic solutions of (2.2) corresponding to the values $\Lambda^{1 / 2}$ and $-\Lambda^{1 / 2}$, where $\Lambda$ is a given positive value, are linearly independent. Hence the index of $\Lambda$ as a characteristic value of (3.12) is not less than the sum of the indices of $\Lambda^{1 / 2}$ and $-\Lambda^{1 / 2}$ as characteristic values of (2.2). We have, therefore, the following theorem:

THEOREM 3.4. The characteristic values of (2.2) are all real and at most denumerably infinite in number. If $\Lambda$ is a characteristic value of (3.12), either $\Lambda^{1 / 2}$ or $-\Lambda^{1 / 2}$ is a characteristic value of (2.2). Conversely, if $\lambda$ is a characteristic value of (2.2), then $\Lambda=\lambda^{2}$ is a characteristic value of (3.12) whose index is equal to the sum of the indices of $\lambda$ and $-\lambda$ as characteristic values of (2.2).

4. Sufficient conditions for the existence of infinitely many characteristic values. One may show by simple examples (see Bliss [3]) that the hypotheses of $\$ 2$ do not imply the existence of infinitely many characteristic values of (2.2). In this section we shall give certain sets of conditions that insure this property. In view of Theorem 3.4 we may assume without loss of generality that $\lambda=0$ is not a characteristic value of $(2.2)$, and the sets $u_{i \kappa},(\kappa=1, \cdots, \rho)$, are missing. If this condition is not true for the original problem it may be attained by a linear change of parameter. We shall make this assumption in the future consideration of this problem. The following general theorem follows from Theorem 3.4 and the extremizing properties of the characteristic values of (3.12) (see, for example, Reid [9] and [11]).

THEOREM 4.1. A system (2.2) satisfying $\left(\mathrm{H}_{1}\right)-\left(\mathrm{H}_{5}\right)$ has an infinity of characteristic values if and only if there are infinitely many arcs $z_{i}=w_{i s}(x)$, $(s=1,2, \cdots)$, satisfying (3.4), (3.5), and such that for each $r$ and arbitrary constants $\left(d_{t}\right) \neq\left(0_{t}\right),(t=1, \cdots, r)$, the arc $w_{i}=w_{i t}(x) d_{t}$ satisfies the condition

$$
\int_{a}^{b} w_{i} K_{i j} w_{j} d x>0
$$

The following hypothesis is a weakened form of a condition used by Bliss in originally defining definitely self-adjoint systems. 
$\left(\mathrm{H}_{6}\right)$ If $g_{i}(x)$ are arbitrary continuous functions on $a b$, and $y_{i}(x)$ is a solution of the system

$$
\mathcal{L}_{i}[y]=B_{i j}(x) g_{i}(x), \quad s_{i}[y]=0,
$$

then $y_{i} S_{i j} y_{j} \equiv 0$ on $a b$ if and only if $y_{i} \equiv 0$.

THEOREM 4.2. If (2.2) satisfies $\left(\mathrm{H}_{1}\right),\left(\mathrm{H}_{2}\right),\left(\mathrm{H}_{3}\right),\left(\mathrm{H}_{5}\right)$, and $\left(\mathrm{H}_{6}\right)$, this system has infinitely many characteristic values.

Hypotheses $\left(\mathrm{H}_{2}\right),\left(\mathrm{H}_{3}\right)$, and $\left(\mathrm{H}_{6}\right)$ are seen to imply $\left(\mathrm{H}_{4}\right)$. Denote by $g_{i s}(x)$, $(s=1,2, \cdots)$, sets of functions such that the sets $B_{i j}(x) g_{j_{s}}(x)$ are linearly independent on $a b$. Such a choice is clearly possible. Now let $y_{i}=y_{i s}(x)$ be a particular solution of (4.2) for $g_{i}=g_{i s}(x)$, and set $w_{i s}(x)=T_{i i}(x) y_{j s}(x)$, $(s=1,2, \cdots)$. By the use of relation (2.5) it may be proved that the functions $z_{i}=w_{i s}(x)$ satisfy (3.4) and (3.5). Furthermore, if $\left(d_{t}\right) \neq\left(0_{t}\right)$, $(t=1, \cdots, r)$, and $y_{i}=y_{i t}(x) d_{t}, w_{i}=w_{i t}(x) d_{t}$ we have

$$
\int_{a}^{b} w_{i} K_{i j} w_{i} d x=\int_{a}^{b} y_{i} S_{i j} y_{j} d x>0
$$

in view of $\left(\mathrm{H}_{6}\right)$. The result of Theorem 4.2 is then a consequence of Theorem 4.1 .

Corollary. If the system (2.2) satisfies hypotheses $\left(\mathrm{H}_{1}\right),\left(\mathrm{H}_{2}\right),\left(\mathrm{H}_{3}\right)$, and $\left|B_{i j}\right| \neq 0$ on ab, then (2.2) has infinitely many characteristic values.

Hypothesis $\left(\mathrm{H}_{6}\right)$ is weaker than the condition originally used by Bliss [2] in defining definitely self-adjoint systems since the extra condition $s_{i}[y]=0$ has been added. The result of Theorem 4.2 is of a somewhat less general character than that originally obtained by Bliss [2], however, since we have assumed $\left(\mathrm{H}_{5}\right)$.

THEOREM 4.3. Suppose that (2.2) satisfies $\left(\mathrm{H}_{1}\right)-\left(\mathrm{H}_{5}\right)$, and that the functions $B_{i j}$ are of class $C^{1}$ on $a b$. Then, if the matrix

$$
\left\|\pi_{i \alpha}(x) T_{k i}^{-1}(x) B_{j k}(x)\right\|
$$

has at some point $x_{0}$ of ab a rank greater than $m$, the system (2.2) has infinitely many characteristic values.

If the functions $B_{i j}$ are of class $C^{\mathbf{1}}$, the functions $\pi_{i \alpha}(x)$ may also be chosen to be of class $C^{1}$, and we shall suppose that these functions are so selected. Now a set $z_{i}$ satisfies (3.4) if and only if there are functions $g_{i}(x)$ such that

$$
\mathcal{X}_{i}[z]=-g_{j}(x) B_{j i}(x) .
$$

In view of $\left(\mathrm{H}_{2}\right)$ and $\left(\mathrm{H}_{3}\right)$ a set of continuous functions $z_{i}(x)$ renders 
$\int_{a}^{b} z_{i} K_{i j} z_{j} d x=0$ if and only if the set $z_{i}$ is of the form $T_{j i}(x) \pi_{j \alpha}(x) f_{\alpha}(x)$. We shall now determine under what conditions a set of this latter form is a solution of (4.4). In view of (2.5), such a set satisfies (4.4) if and only if

$$
\mathcal{L}_{i}\left[\pi_{\alpha} f_{\alpha}\right] \equiv \pi_{i \alpha} f_{\alpha}^{\prime}+\mathcal{L}_{i}\left[\pi_{\alpha}\right] f_{\alpha}=-g_{j} B_{j k} T_{k i}^{-1} .
$$

The functions $\mathcal{L}_{i}\left[\pi_{\alpha}\right]$ are continuous since $\pi_{i \alpha}(x)$ are of class $C^{1}$. Now, in view of the hypotheses of the theorem, there is an interval $a_{1} b_{1}$ containing $x_{0}$ and integers $1 \leqq i_{1}<i_{2}<\cdots<i_{p} \leqq n, 1 \leqq j_{1}<j_{2}<\cdots<j_{p-m} \leqq n, p>m$, such that the determinant $\left|\pi_{i_{q^{\alpha}}} T_{\boldsymbol{k}_{q}}^{-1} B_{j_{h} k}\right|,(q=1, \cdots, p ; h=1, \cdots, p-m)$, is different from zero on $a_{1} b_{1}$. If $g_{j}=0$ for $j \neq j_{h},(h=1, \cdots, p-m)$, then on $a_{1} b_{1}$ there are continuous functions $D_{\alpha \beta}(x), E_{h \beta}(x)$ such that

$$
\begin{aligned}
& f_{\alpha}^{\prime}=D_{\alpha \beta} f_{\beta}, \\
& g_{j_{h}}=E_{h \beta} f_{\beta} .
\end{aligned}
$$

Let $f_{\alpha}=f_{\alpha \gamma}(x),(\gamma=1, \cdots, m)$ be a fundamental set of solutions of (4.6) on $a_{1} b_{1}$. Suppose that $g_{j}^{*}(x)$ is a set of functions such that $g_{j}^{*}=0$ for $j \neq j_{h}$, and the set $g_{j_{h}}^{*}$ is linearly independent of the $m$ sets of functions $g_{i_{h}}=E_{h \beta} f_{\beta \gamma}$ on $a_{1} b_{1}$. It follows that if $z_{i}=w_{i}$ is the solution of

$$
\mathcal{H}_{i}[z]=-g_{i}^{*} B_{j i}, \quad t_{i}[z]=0,
$$

then $w_{i}$ is not of the form $T_{j i} \pi_{j \alpha} f_{\alpha}$ on $a_{1} b_{1}$, and hence $\int_{a}^{b} w_{i} K_{i j} w_{j} d x>0$. Now one may choose an infinity of sets $g_{f s}^{*}(x),(s=1, \cdots)$, such that if $r$ is a given integer and $\left(d_{t}\right) \neq\left(0_{t}\right)(t=1, \cdots, r)$, then the set $g_{j}^{*}=g_{j t}^{*}(x) d_{t}$ satisfies the conditions described above for the set $g_{j}^{*}$. If $z_{i}=w_{i s}$ are the corresponding solutions of (4.8), we have that the set $w_{i s},(s=1, \cdots)$, satisfies the conditions of Theorem 4.1, and hence (2.2) has infinitely many characteristic values.

We have, in particular, the following result:

Corollary. Suppose that (2.2) satisfies $\left(\mathrm{H}_{1}\right)-\left(\mathrm{H}_{5}\right)$, the functions $B_{i j}$ are of class $C^{1}$ on ab, and $m<n-m$. Then this system has infinitely many characteristic values.

To establish this corollary, one need only note that the rank of (4.3) is not less than the greater of the two values $m$ and $n-m$.

5. A special boundary problem. Consider the differential equations

$$
\begin{aligned}
& \eta_{\sigma}^{\prime}=\mathcal{A}_{\sigma \tau}(x) \eta_{\tau}+\mathcal{B}_{\sigma \tau}(x) \zeta_{\tau}, \quad \sigma, \tau=1, \cdots, N, \\
& \zeta_{\sigma}^{\prime}=\mathcal{C}_{\sigma \tau}(x) \eta_{\tau}-\mathcal{A}_{\tau \sigma}(x) \zeta_{\tau}-\lambda K_{\sigma \tau}(x) \eta_{\tau},
\end{aligned}
$$

where $\left\|\mathcal{B}_{\sigma \tau}\right\|,\left\|\mathcal{C}_{\sigma \tau}\right\|,\left\|K_{\sigma \tau}\right\|$ are symmetric. The coefficients in (5.1) are realvalued and continuous on $a b$, and $u_{\sigma} K_{\sigma \tau} u_{\tau}>0$ on $a b$ for $\left(u_{\sigma}\right) \neq\left(0_{\sigma}\right)$. Associated with (5.1) we consider boundary conditions 


$$
\begin{aligned}
s_{\gamma}[\eta, \zeta] \equiv a_{\gamma \tau}^{1} \eta_{\tau}(a)-b_{\gamma \tau}^{1} \zeta_{\tau}(a)+a_{\gamma \tau}^{2} \eta_{\tau}(b)+b_{\gamma \tau}^{2} \zeta_{\tau}(b) & =0, \\
\gamma & =1, \cdots, 2 N,
\end{aligned}
$$

where these conditions are linearly independent, and where the matrix $\left\|a_{\gamma \tau}^{1} b_{\delta \tau}^{1}+a_{\gamma \tau}^{2} b_{\delta \tau}^{2}\right\|, \quad(\gamma, \delta=1, \cdots, 2 N)$, is symmetric. We shall also suppose that there is no non-identically vanishing solution $\eta_{\sigma}, \zeta_{\sigma}$ of (5.1), (5.2) with $\eta_{\sigma} \equiv 0,(\sigma=1, \cdots, N)$, on $a b$.

The system (5.1), (5.2) is self-adjoint with respect to the transformation matrix

$$
\|T\|=\left\|\begin{array}{rr}
0_{\sigma \tau} & \delta_{\sigma \tau} \\
-\delta_{\sigma \tau} & 0_{\sigma \tau}
\end{array}\right\|
$$

Under the above conditions,

$$
\|B\|=\left\|\begin{array}{cc}
0_{\sigma \tau} & 0_{\sigma \tau} \\
-K_{\sigma \tau} & 0_{\sigma \tau}
\end{array}\right\|, \quad\|S\|=\left\|\begin{array}{cc}
K_{\sigma \tau} & 0_{\sigma \tau} \\
0_{\sigma \tau} & 0_{\sigma r}
\end{array}\right\|, \quad\|K\|=\left\|\begin{array}{cc}
0_{\sigma \tau} & 0_{\sigma \tau} \\
0_{\sigma \tau} & K_{\sigma \tau}
\end{array}\right\|,
$$

and (5.1), (5.2) satisfies $\left(\mathrm{H}_{1}\right)-\left(\mathrm{H}_{6}\right)$ of $\$ 2$. We may, therefore, assume without loss of generality that $\lambda=0$ is not a characteristic value, and for simplicity we shall make this assumption.

If we assume in addition that $\left\|\mathcal{B}_{\sigma \tau}\right\|$ has constant rank $N-q,(0 \leqq q<N)$, on $a b$, this system is equivalent to a boundary problem associated with the second variation of a problem of Bolza in the calculus of variations which is normal and non-singular, but which does not necessarily satisfy the Clebsch condition. If $K_{\sigma \tau}=\delta_{\sigma \tau}$, we have the problem which $\mathrm{Hu}$ [5] considered, and for which he proved the existence of infinitely many characteristic values. His method of proof is closely related to that previously used by Bliss [2] in treating definitely self-adjoint problems. In the following, we shall not assume that $\left\|\mathcal{B}_{\sigma r}\right\|$ has constant rank $N-q$, but simply that all the $\mathcal{B}_{\sigma r}$ are not identically zero on $a b$.

The system adjoint to (5.1), (5.2) is

$$
\begin{aligned}
u_{\sigma}^{\prime}+u_{\tau} A_{\tau \sigma}+v_{\tau} \mathcal{C}_{\tau \sigma} & =\lambda v_{\tau} K_{\tau \sigma}, \\
v_{\sigma}^{\prime}+u_{\tau} \mathbb{B}_{\tau \sigma}-v_{\tau} \mathcal{C} A_{\sigma \tau} & =0, \\
b_{\gamma \tau}^{1} u_{\tau}(a)+a_{\gamma \tau} v_{\tau}(a)-b_{\gamma \tau}^{2} u_{\tau}(b)+a_{\gamma \tau}^{2} v_{\tau}(b) & =0, \quad \gamma=1, \cdots, 2 N .
\end{aligned}
$$

The condition (3.4) then becomes, in terms of $\left(z_{i}\right)=\left(u_{\sigma}, v_{\sigma}\right)$,

$$
v_{\sigma}^{\prime}+u_{\tau} \mathbb{B}_{\tau \sigma}-v_{\tau} \mathcal{A}_{\sigma \tau}=0, \quad \sigma=1, \cdots, N,
$$

and the integral of (3.7) reduces to

$$
\int_{a}^{b} v_{\sigma} K_{\sigma \tau} v_{\tau} d x .
$$


Let $a_{1} b_{1}$ be a subinterval of $a b$ on which all the $\mathcal{B}_{\sigma \tau}$ are not identically zero, and by $u_{\tau}=u_{r v},(\nu=1, \cdots, N+1)$, sets of functions of class $C^{1}$ on $a_{1} b_{1}$, with $u_{\tau \nu}\left(a_{1}\right)=0=u_{\tau \nu}\left(b_{1}\right)$, and such that the $N+1$ sets $u_{\tau \nu} \mathbb{B}_{\tau \sigma},(\nu=1, \cdots, N+1)$, are linearly independent on this interval. Let $v_{\tau}=v_{\tau \nu}(x)$ be the solution of (5.5) for $u_{\tau}=u_{\tau \nu}$, and satisfying the initial condition $v_{\tau \nu}\left(a_{1}\right)=0$. Clearly the sets $v_{\tau}=v_{\tau \nu}(x),(\nu=1, \cdots, N+1)$, are linearly independent on $a_{1} b_{1}$. Now define $v_{\tau}=v_{\tau 1}(x) d_{1}+\cdots+v_{\tau+1}(x) d_{N+1}$, where the constants $d_{t},(t=1, \cdots, N+1)$, are not all zero and such that $v_{\tau}\left(b_{2}\right)=0$. If the functions $u_{\tau}, v_{\tau}$ thus defined on $a_{1} b_{1}$ are extended to the whole of $a b$ by defining them as zero on the remainder of $a b$, then $\left(z_{i}\right)=\left(u_{\tau}, v_{\tau}\right)$ is an admissible arc for the above defined problem, and the corresponding integral (5.6) is positive.

Now let $\Delta_{s},(s=1,2, \cdots)$, be a denumerable set of non-overlapping subintervals of $a_{1} b_{1}$, and relative to $\Delta_{s}$ define a set of functions $\left(z_{i}\right) \equiv\left(w_{i s}\right) \equiv\left(u_{r s}, v_{\tau s}\right)$ in the manner described above. This set $\left(w_{i s}\right)$ is seen to satisfy the conditions of Theorem 4.1; hence the system (5.1) has a denumerable infinity of characteristic values.

6. Oscillation and comparison theorems. The following comparison theorem is a consequence of Theorem 5.5 of Reid [11].

THEOREM 6.1. Suppose that the two boundary value problems

$$
\begin{array}{ll}
\mathcal{L}_{i}[y]=\lambda B_{i j} y_{j}, & s_{i}[y] \equiv M_{i j} y_{j}(a)+N_{i j} y_{j}(b)=0, \\
\mathcal{L}_{i}[y]=\lambda B_{i j} y_{j}, & s_{i}^{*}[y] \equiv M_{i j}^{*} y_{j}(a)+N_{i j}^{*} y_{j}(b)=0,
\end{array}
$$

are self-adjoint with respect to the same transformation matrix $\left\|T_{i j}\right\|$, that each of these problems satisfies $\left(\mathrm{H}_{1}\right)-\left(\mathrm{H}_{6}\right)$, and $\lambda=0$ is not a characteristic value for either of these problems. For arbitrary values $L>0$, let $V_{L}\left[W_{L}\right]$ denote the number of characteristic values $\lambda$ of (6.1) such that $|\lambda|<L[|\lambda| \leqq L]$. The numbers $V_{L}^{*}$, $W_{L}^{*}$ are defined for (6.2) in an analogous manner. If the matrix

$$
\left\|\begin{array}{ll}
M_{i j} & N_{i j} \\
M_{i j}^{*} & N_{i j}^{*}
\end{array}\right\|
$$

has rank $n+h$, then for arbitrary $L>0$ we have $\left|V_{L}-V_{L}^{*}\right| \leqq h,\left|W_{L}-W_{L}^{*}\right| \leqq h$.

We shall say that a point $x^{\prime}$ on $a<x^{\prime} \leqq b$ is a conjugate point of $x=a$ relative to the differential equations

$$
\mathcal{L}_{i}[y]=\lambda B_{i j}(x) y_{j}
$$

for a value $\lambda$, if, using the definition of Reid [11], $\S 6$, the point $x^{\prime}$ is a conjugate point of $x=a$ relative to the differential equations of (3.12) for the value $\Lambda=\lambda^{2}$. It is to be remarked that the hypotheses of $\$ 2$ do not in general 
imply that the order of abnormality of the differential equations of (3.12) on subintervals $a b^{\prime}$ is constant for $a<b^{\prime} \leqq b$. Hence, as pointed out in Reid [11], in order to obtain the usual oscillation theorem it is necessary to use the extended definition of conjugate point there introduced. As a result of Theorem 3.4 above, and the oscillation and comparison theorems for the system (3.12) (see Reid [11], $\$ 5,6$, and 7), we have the following result:

THEOREM 6.2. Suppose that the system (6.1) satisfies hypotheses $\left(\mathbf{H}_{1}\right)-\left(\mathbf{H}_{5}\right)$ and that $\lambda=0$ is not a characteristic value of this problem. Then for arbitrary values $\lambda \neq 0$ there are on the interval $a<x<b$ at least $V_{|\lambda|}-n$ and at most $V_{|\lambda|}$ conjugate points of $x=$ a relative to (6.3) for the given $\lambda$.

In case the following additional assumption is satisfied, the conjugate points are given by the zeros of a certain determinant.

$\left(\mathrm{H}_{7}\right)$ If $b^{\prime}$ is an arbitrary value on $a<b^{\prime} \leqq b$, there is no solution of $\mathcal{L}_{i}[y]=0$ satisfying $B_{i j}(x) y_{j}(x) \equiv 0$ on $a b^{\prime}$ except the identically vanishing solution $y_{i} \equiv 0$.

The statement $\left(\mathrm{H}_{7}\right)$ is the phrasing in terms of the system (6.3) of the condition that the differential equations of (3.12) are normal on every subinterval $a b^{\prime}$. In this case the conjugate points of $x=a$ relative to (3.12) for a value $\Lambda$ are the zeros of $\left|z_{i j}(x)\right|$, where

$$
z_{i}=z_{i j}(x), \zeta_{i}=\zeta_{i j}(x), \quad j=1, \cdots, n,
$$

are linearly independent solutions of the differential equations of (3.12) such that

$$
z_{i j}(a)=0, \quad i, j=1, \cdots, n .
$$

THEOREM 6.3. Suppose that the system (6.1) satisfies $\left(\mathrm{H}_{7}\right)$ in addition to the hypotheses of Theorem 6.2. If $y_{i}=y_{i j}(x: \lambda),(j=1, \cdots, n)$, denote the solutions of (6.3) for which $y_{i j}(a: \lambda)=\delta_{i j}$, then for arbitrary values $\lambda \neq 0$ the determinant

$$
\left|y_{i j}(x: \lambda)-y_{i j}(x:-\lambda)\right|
$$

has on $a<x<b$ at least $V_{|\lambda|}-n$ and at most $V_{|\lambda|}$ zeros, each zero counted a number of times equal to its index for the system of linear homogeneous equations whose coefficients are the rows of the determinant (6.4).

Theorem 6.3 is a ready consequence of the fact that

$$
z_{i j} \equiv(1 / \lambda) T_{k i}(x)\left[y_{k j}(x: \lambda)-y_{k j}(x:-\lambda)\right], \quad \zeta_{i j} \equiv y_{i j}(x: \lambda)+y_{i j}(x:-\lambda)
$$

is a linearly independent set of solutions of the differential equations of (3.12) for $\Lambda=\lambda^{2}$ such that $z_{i j}(a)=0,(i, j=1, \cdots, n)$. 


\section{BIBLIOGRAPHY}

1. Birkhoff and Hestenes, Natural isoperimetric conditions in the calculus of variations, Duke Mathematical Journal, vol. 1 (1935), pp. 198-286.

2. Bliss, $A$ boundary value problem for a system of ordinary linear differential equations of the first order, these Transactions, vol. 28 (1926), pp. 561-584.

3. Bliss, Definitely self-adjoint boundary value problems, these Transactions, vol. 44 (1938), pp. 413-428.

4. Hölder, Die Lichtensteinsche Method fïr die Entwicklung der zweiten Variation, angewandt auf das Problem von Lagrange, Prace Matematyczno-Fizyczne, vol. 42 (1935), pp. 307-346.

5. Hu, The problem of Bolza and its accessory boundary value problem, Contributions to the Calculus of Variations 1931-1932, The University of Chicago Press, pp. 361-443.

6. Ince, Ordinary Differential Equations, London, 1927.

7. Morse, Sufficient conditions in the problem of Lagrange with variable end conditions, American Journal of Mathematics, vol. 53 (1931), pp. 517-546.

8. Morse, The Calculus of Variations in the Large, American Mathematical Society Colloquium Publications, vol. 18, 1934.

9. Reid, $A$ boundary value problem associated with the calculus of variations, American Journal of Mathematics, vol. 54 (1932), pp. 769-790.

10. Reid, Analogues of the Jacobi condition for the problem of Mayer in the calculus of variations, Annals of Mathematics, (2), vol. 35 (1934), pp. 836-848.

11. Reid, An integro-differential boundary value problem, American Journal of Mathematics, vol. 60 (1938), pp. 257-292.

12. Wiggin, $A$ boundary value problem of the calculus of variations, Contributions to the Calculus: of Variations 1933-1937, The University of Chicago Press, pp. 245-275.

UNIVERSTTY OF ChicAgo, Chicago, Ill. 